\title{
Consequences of natural perturbations in the human plasma proteome
}

Benjamin B. Sun ${ }^{1}$, Joseph C. Maranville ${ }^{2} *$ James E. Peters ${ }^{1,3}$, David Stacey ${ }^{1}$, James R. Staley ${ }^{1}$, James Blackshaw ${ }^{1}$, Stephen Burgess ${ }^{1,4}$, Tao Jiang ${ }^{1}$, Ellie Paige ${ }^{1}$, Praveen Surendran ${ }^{1}$, Clare Oliver-Williams ${ }^{1}$, Mihir A. Kamat ${ }^{1}$, Bram P. Prins ${ }^{1}$, Sheri K. Wilcox ${ }^{5}$, Erik S. Zimmerman $^{5}$, An $\mathrm{Chi}^{2}$, Narinder Bansal ${ }^{1,6}$, Sarah L. Spain ${ }^{7}$, Angela M. Wood ${ }^{1}$, Nicholas W. Morrell $^{8}$, John R. Bradley9, Nebojsa Janjic ${ }^{5}$, David J. Roberts ${ }^{10,11}$, Willem H. Ouwehand $^{3,12,13,14,15}$, John A. Todd ${ }^{16}$, Nicole Soranzo ${ }^{3,12,14,15}$, Karsten Suhre ${ }^{17}$, Dirk S. Paul ${ }^{1}$, Caroline S. Fox ${ }^{2}$, Robert M. Plenge ${ }^{2}$, John Danesh ${ }^{1,3,14,15}$, Heiko Runz ${ }^{2 *}$, Adam S. Butterworth $^{1,15 *}$

1. MRC/BHF Cardiovascular Epidemiology Unit, Department of Public Health and Primary Care, University of Cambridge, Cambridge CB1 8RN, UK.

2. MRL, Merck \& Co., Inc., Kenilworth, New Jersey, USA.

3. British Heart Foundation Cambridge Centre of Excellence, Division of Cardiovascular Medicine, Addenbrooke's Hospital, Cambridge CB2 0QQ, UK.

4. MRC Biostatistics Unit, University of Cambridge, Cambridge CB2 0SR, UK.

5. SomaLogic Inc., Boulder, Colorado 80301, USA.

6. Perinatal Institute, Birmingham B15 3BU, UK.

7. Wellcome Trust Sanger Institute, Wellcome Trust Genome Campus, Hinxton, Cambridge CB10 1RQ, UK.

8. Division of Respiratory Medicine, Department of Medicine, University of Cambridge, Cambridge CB2 0QQ, UK.

9. NIHR Cambridge Biomedical Research Centre / BioResource, Cambridge University Hospitals, Cambridge CB2 0QQ, UK.

10. National Health Service (NHS) Blood and Transplant and Radcliffe Department of Medicine, NIHR Oxford Biomedical Research Centre, University of Oxford, John Radcliffe Hospital, Oxford OX3 9DU, UK.

11. BRC Haematology Theme and Department of Haematology, Churchill Hospital, Oxford OX3 7LE, UK.

12. Department of Haematology, University of Cambridge, Cambridge Biomedical Campus, Long Road, Cambridge CB2 0PT, UK.

13. National Health Service (NHS) Blood and Transplant, Cambridge Biomedical Campus, Cambridge CB2 0PT, UK.

14. Department of Human Genetics, Wellcome Trust Sanger Institute, Wellcome Trust Genome Campus, Hinxton, Cambridge CB10 1RQ, UK.

15. NIHR Blood and Transplant Research Unit in Donor Health and Genomics, Department of Public Health and Primary Care, University of Cambridge, Cambridge CB1 8RN, UK.

16. JDRF/Wellcome Trust Diabetes and Inflammation Laboratory, Wellcome Trust Centre for Human Genetics, Nuffield Department of Medicine, NIHR Oxford Biomedical Research Centre, University of Oxford, Oxford OX3 7BN, UK.

17.Department of Physiology and Biophysics, Weill Cornell Medicine - Qatar, PO 24144 Doha, Qatar.

* These authors contributed equally to this work.

Corresponding authors: asb38@medschl.cam.ac.uk (A.S.B.),jd292@medschl.cam.ac.uk (J.D.) 


\section{Abstract}

49 Proteins are the primary functional units of biology and the direct targets of most drugs, yet

50 there is limited knowledge of the genetic factors determining inter-individual variation in

51 protein levels. Here we reveal the genetic architecture of the human plasma proteome, testing

5210.6 million DNA variants against levels of 2,994 proteins in 3,301 individuals. We identify

53 1,927 genetic associations with 1,478 proteins, a 4-fold increase on existing knowledge,

54 including trans associations for 1,104 proteins. To understand consequences of perturbations

55 in plasma protein levels, we introduce an approach that links naturally occurring genetic

56 variation with biological, disease, and drug databases. We provide insights into pathogenesis

57 by uncovering the molecular effects of disease-associated variants. We identify causal roles

58 for protein biomarkers in disease through Mendelian randomization analysis. Our results

59 reveal new drug targets, opportunities for matching existing drugs with new disease

60 indications, and potential safety concerns for drugs under development. 


\section{Introduction}

62 Plasma proteins play key roles in biological processes such as signalling, transport, growth,

63 repair, and defence against infection. They are frequently dysregulated in disease and are the 64 targets of many drugs. Detailed characterisation of the genetic factors that determine interindividual protein variability will, therefore, furnish both fundamental and applied insights ${ }^{1}$. Despite evidence of the heritability of plasma protein abundance ${ }^{2}$, systematic genome-wide study of such 'protein quantitative trait loci' (pQTLs) has been constrained by inability to measure large numbers of proteins reliably in large numbers of individuals ${ }^{1,3-5}$.

Here we combine several technical and conceptual advances to create and interrogate a rich genetic atlas of the human plasma proteome. First, we use a markedly expanded version of an aptamer-based multiplex protein assay $\left(\right.$ SOMAscan) $^{6}$ to quantify 3,620 plasma proteins in 3,301 healthy individuals, representing several-fold increases both in breadth of protein panel and in cohort size. Second, we exploit improvements in genotype imputation panels to achieve 10-fold denser genotypic coverage than in previous proteomic studies ${ }^{7}$. Third, we draw on studies with multi-dimensional data (e.g., transcriptomics and clinical phenotypes) and on new bioinformatics tools to help understand mechanisms and clinical consequences of perturbations in protein pathways ${ }^{8}$. Fourth, we use two-sample "Mendelian randomization" techniques to evaluate the causal relevance of protein biomarkers to disease ${ }^{9}$. Finally, we cross-reference genomic-proteomic information with disease and drug databases to identify and prioritise therapeutic targets.

Our study characterises the genetic architecture of the human plasma proteome, identifying 1,927 genotype-protein associations, including trans-associated loci for 1,104 proteins that provide key insights into protein regulation. More than 150 pQTLs overlap with disease 
susceptibility loci, elucidating the molecular effects of disease-associated variants. We find strong evidence to support causal roles in disease for several protein pathways, highlighting novel therapeutic targets as well as potential safety concerns for drugs in development.

\section{Genetic architecture of the plasma proteome}

After stringent quality control, we performed genome-wide testing of 10.6 million autosomal variants against levels of 2,994 plasma proteins in 3,301 healthy European-ancestry individuals (Methods, Extended Data Figure 1). Genotypes were measured using the Affymetrix Axiom UK Biobank array and imputed against a combined 1000 Genomes and UK10K reference panel. Protein levels were measured using the SOMAscan assay. We evaluated the robustness of protein measurements in several ways (Methods, $\underline{\text { Supplementary }}$ Note). Measurements in replicate samples were highly consistent; the median coefficient of variation across all proteins was 0.064 (interquartile range 0.049-0.092). We also showed temporal consistency in protein levels in samples obtained two years apart from the same individuals, reproduced known associations with non-genetic factors, and verified selected protein measurements with multiple different assay methods.

We found 1,927 genome-wide significant $\left(p<1.5 \times 10^{-11}\right)$ associations between 1,478 proteins and 764 genomic regions (Figure 1a, Supplementary Table 1, Supplementary Video 1), with $89 \%$ of pQTLs reported here for the first time. Of these 764 regions, $502(66 \%)$ had cis associations only, $228(30 \%)$ had trans associations only and $34(4 \%)$ had both cis and trans. $95 \%$ and $87 \%$ of our cis pQTL variants were located within $200 \mathrm{~Kb}$ and $100 \mathrm{~Kb}$, respectively,

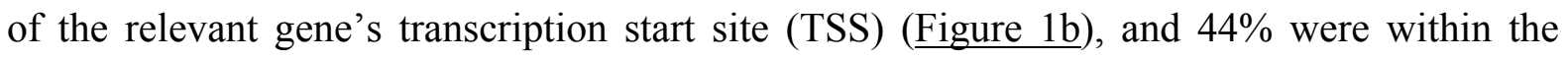
gene itself. The $p$-values for cis pQTL associations increased with increasing distance from the TSS, mirroring findings for cis expression QTLs (eQTLs) in transcriptomic studies ${ }^{10,11}$. 
111 Of the proteins for which we identified a pQTL, 88\% had either cis $(\mathrm{n}=374)$ or trans $(\mathrm{n}=925)$

112 associations only, while $12 \%(n=179)$ had both. The majority of significantly associated

113 proteins $(75 \% ; n=1,113)$ had a single pQTL, while $20 \%$ had two and $5 \%$ had more than two

114 (Figure 1c). To detect multiple signals at the same locus we used stepwise conditional 115 analysis, identifying 2,658 conditionally significant associations (Supplementary Table 2). Of

116 the 1,927 locus-protein associations, 414 (21\%) had multiple conditionally significant signals

117 (Figure 1d), of which 255 were cis.

119 Genetic variants that change the protein structure may result in apparent pQTLs due to 120 altered aptamer binding rather than true quantitative differences in protein levels. Using 121 bioinformatic approaches to evaluate the extent to which cis pQTLs might reflect technical effects (Methods) we found that $91 \%$ of pQTLs were unlikely to be influenced by differential aptamer binding (Supplementary Tables 1 and 3$)$. Furthermore, in sub-studies that involved immunoassays, we found strongly concordant genotype-protein associations (Supplementary Note). Even where apparent pQTLs do arise from alternative protein structure that may affect aptamer binding rather than differences in protein abundance, such signals may be functionally significant.

129 The median variation in protein levels explained by our pQTLs was 5.8\% (in-sample 130 estimate; interquartile range: 2.6\%-12.4\%, Figure 1e). For 193 proteins, however, genetic 131 variants explained more than $20 \%$ of the variation, such as for teratocarcinoma-derived growth factor $1(65 \%)$ and haptoglobin $(55 \%)$. We found a strong inverse relationship between effect size and minor allele frequency (MAF) (Figure 1f), consistent with previous

134 genome-wide association studies (GWAS) of quantitative traits ${ }^{12-14}$. We found 25 135 associations with rare $(\mathrm{MAF}<1 \%$ ) variants and a further 207 associations with low- 
136 frequency (MAF 1-5\%) variants. Of the 31 strongest pQTLs (per-allele effect size $>1.5$

137 standard deviations), 25 were rare or low-frequency variants.

139 pQTLs were strongly enriched for missense variants $(p<0.0001)$ and for location in $3^{\text {' }}$ 140 untranslated $(p=0.0025)$ or splice regions $(p=0.0004)$ (Figure 1g, Extended Data Figure 2).

141 To assess whether pQTLs were enriched within regulatory elements from a wide range of cell 142 types and tissues ${ }^{15-17}$, we used GARFIELD ${ }^{18}$ (Methods). We found strong ( $\geqslant 3$-fold, $\left.143 p<5 \times 10^{-5}\right)$ enrichment of pQTLs in blood cells - unsurprisingly given our use of plasma - at

144 features indicative of transcriptional activation (Extended Data Figure 3, Supplementary

145 Table 4). We also found enrichment at hepatocyte regulatory elements, consistent with the 146 liver's role in producing many secreted proteins.

148 Overlap of loci for gene expression and protein levels

149 A fundamental biological question is the extent to which genetic associations with plasma

150 protein levels are driven by effects at the transcription level, rather than other mechanisms,

151 such as altered protein clearance or cleavage of membrane receptor proteins from the cell surface. We therefore cross-referenced our cis pQTLs with a wide range of publicly available eQTL studies involving $>30$ tissues or cell types (Supplementary Table 5) using

154 PhenoScanner $^{8} .40 \%(n=224)$ of cis pQTLs had an association with expression of the same 155 gene in at least one tissue (Supplementary Table 6), consistent with similar comparisons 156 within lymphoblastoid cell lines (LCLs) ${ }^{19}$. The greatest overlaps were found in whole blood 157 ( $\mathrm{n}=117)$, liver $(\mathrm{n}=70)$ and LCLs $(\mathrm{n}=52)$, consistent with biological expectation, but also likely driven by the larger sample sizes for expression studies of these cell types. Cis pQTLs were significantly enriched $(p<0.0001)$ for eQTLs for the corresponding gene (mean $20 \%$ versus

$1602 \%$ in a background permuted set; Methods, Supplementary Table 7). To address the 
161 converse (i.e., to what extent do eQTLs translate into pQTLs), we used a subset of wellpowered eQTL studies in relevant tissues (whole blood, LCLs, liver and monocytes ${ }^{20-23}$ ). Of

163 the strongest cis eQTLs $\left(p<1.5 \times 10^{-11}\right), 12.2 \%$ of those in whole blood were also cis pQTLs, $16421.3 \%$ for LCLs, $14.8 \%$ for liver and $14.7 \%$ for monocytes.

166 Comparisons between eQTL and pQTL studies have inherent limitations due to differences in 167 the tissues, sample sizes and technological platforms used. Moreover, plasma protein levels 168 may not reflect levels within tissues or cells. Nevertheless, our data suggest that plasma 169 protein abundance is often, but not exclusively, driven by regulation of mRNA. Our finding 170 that pQTLs are enriched in gene regulatory regions supports the notion that transcription has a major role. Cis pQTLs without corresponding cis eQTLs may be underpinned by effects not captured at the mRNA level, such as differences in protein stability, degradation, binding, secretion, or clearance from circulation.

\section{Using trans pQTLs to illuminate biological pathways}

176 Trans pQTLs are particularly useful for understanding biological relationships between

177 proteins if a causal gene at the trans-associated genetic locus can be identified. To this end, we used a combination of databases of molecular pathways and protein-protein interaction networks, and functional genomic data that include variant annotation, eQTL and

180 chromosome conformation capture, to link trans-associated variants to potential causal genes

181 (Methods, Supplementary Table 8, Extended Data Figure 4). Of the 764 protein-associated regions, 262 had trans associations with 1,104 proteins. We replicated previously reported trans associations including TMPRSS6 with transferrin receptor protein $1^{24}$ and SORT1 with granulins $^{25}$. Most (82\%) trans loci were associated with fewer than four proteins. However, 
186 Figure 5), including well-known pleiotropic loci (e.g., $A B O, C F H, A P O E, K L K B 1)$ and loci

187 associated with many correlated proteins (e.g., the locus containing the transcription factor gene, ZFPM2).

190 We identified a number of novel associations with strong biological plausibility

191 (Supplementary Table 9, Supplementary Note). Growth differentiation factor 8 (GDF8, more commonly known as myostatin) provides one such example. Because insufficient myostatin has been shown to result in excessive muscle growth ${ }^{26}$, myostatin inhibition has emerged as a

194 promising therapeutic strategy for treatment of conditions characterised by muscle weakness, 195 such as muscular dystrophy ${ }^{27}$. We identified a common allele (rs11079936:C) near 196 WFIKKN2 that is associated in trans with lower levels of plasma GDF11/8 $\left(p=7.9 \times 10^{-12}\right)$, as 197 well as in cis with lower levels of plasma WFIKKN2 $\left(p=6.9 \times 10^{-136}\right.$, Supplementary Table 1 ,

198 Figure 2). The trans association attenuated completely upon adjustment for levels of 199 WFIKKN2 $(p=0.7)$, while the cis association remained significant after adjustment for $200 \operatorname{GDF} 11 / 8\left(p=7.2 \times 10^{-113}\right)$, suggesting that WFIKKN2 regulates GDF11/8. This observation is supported by in vitro evidence suggesting that WFIKKN2 has high affinity for GDF8 and GDF $11^{28}$. This finding illustrates how delineation of causal genes at trans-associated loci may help identify additional drug targets in pathways known to be relevant to disease.

\section{Identifying causal pathways underlying disease susceptibility loci}

206 GWAS have identified thousands of loci associated with common diseases, but the pathways

207 by which most variants influence disease susceptibility await discovery. To identify 208 intermediate links between genotype and disease, we overlapped pQTLs with disease209 associated genetic variants identified through GWAS $\left(p<5 \times 10^{-8}\right) .152$ of our pQTLs were 210 strongly correlated $\left(r^{2} \geqslant 0.8\right)$ with variants significantly associated with disease 
211 (Supplementary Table 10), including 38 with cis associations, 109 with trans associations

212 and 5 with both. In the examples below, we illustrate how our findings provide novel insights

213 spanning a wide range of disease domains including autoimmunity, cancer and

214 cardiovascular disease.

216 Our trans pQTL data implicate previously unsuspected proteins as mediators through which 217 genetic loci exert their influence on disease risk. For example, GWAS have identified a 218 missense allele (rs3197999:A, p.Arg703Cys) in MST1 on chromosome 3 that increases risk 219 of inflammatory bowel disease (IBD) (Figure 3) $)^{29,30}$, and decreases plasma MST1 levels ${ }^{31}$. 220 We show that this polymorphism acts in trans to reduce abundance of BLIMP1, encoded by $221 \quad$ PRDM1 on chromosome 6 (Figure 3, Supplementary Table 1). BLIMP1 is a transcriptional master regulator that plays a critical role in the terminal differentiation of immune cells. Intriguingly, there is another IBD association signal in the intergenic region adjacent to

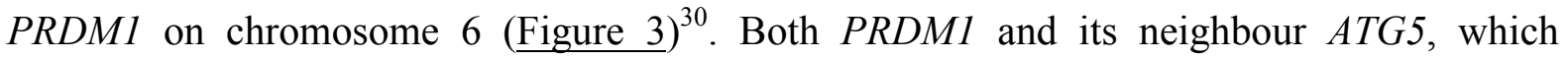
encodes a protein involved in autophagy, are plausible candidate genes at this locus. Our data implicate BLIMP1 as a previously unidentified mediator of the IBD association in MST1 on chromosome 3. In addition, our results provide indirect support for the hypothesis that PRDM1 is the causal gene underlying the chromosome 6 association.

230 We next show how pQTL data can elucidate pathogenic mechanisms. Anti-neutrophil 231 cytoplasmic antibody-associated vasculitis (AAV) is an autoimmune disease characterised by autoantibodies to the neutrophil proteases proteinase-3 (PR3) or myeloperoxidase (MPO).

233 Clinico-pathological features mirror antibody specificity, with granulomatous inflammation

234 typically correlating with anti-PR3 antibodies (PR3+ AAV). GWAS have identified signals 235 in PRTN3 (encoding PR3) and SERPINA1 (encoding alpha1-antitrypsin, an inhibitor of PR3) 
236 specific to PR3+ $\mathrm{AAV}^{32}$. We identified a cis pQTL immediately upstream of PRTN3

237 (Supplementary Table 1). By linking the risk allele at PRTN3 to higher plasma levels of the

238 autoantigen PR3, our data strongly suggest a pathogenic role of anti-PR3 antibodies in this 239 disease.

241 The vasculitis risk allele at the SERPINA1 locus (rs28929474:T, also known as the ' $Z$ ' allele) is a missense variant (p.Glu366Lys) which results in defective secretion of alpha1antitrypsin. We found that the $\mathrm{Z}$ allele was not only associated with lower plasma alpha1antitrypsin, but was also a pQTL "hotspot" associated with 13 proteins using our conservative significance threshold $\left(p<1.5 \times 10^{-11}\right)$ (Figure 4) and 19 at $p<5 \times 10^{-8}$. This finding illustrates how a single mutation can lead to widespread perturbation of downstream proteins. One of these proteins was NCF2 (neutrophil cytosolic factor 2), which plays a key role in the neutrophil oxidative burst. Mutations in $N C F 2$ can result in a rare condition known as chronic granulomatous disease, which like PR3+ AAV exhibits granulomatous inflammation. Our results suggest NCF2 may mediate this characteristic feature of PR3+ AAV.

\section{Causal evaluation of candidate proteins in disease}

253 Association of plasma protein levels with disease does not necessarily imply causation. To

254 help establish causality, we employed the principle of Mendelian randomization (MR) ${ }^{33}$. In contrast with observational studies, which are liable to confounding and/or reverse causation, MR analysis is akin to a "natural" randomised controlled trial, exploiting the random allocation of alleles at conception (Extended Data Figure 6). Consequently, if a genetic variant that specifically influences levels of a protein is also associated with disease risk, then it provides strong evidence of the protein's causal role. For example, serum levels of PSP-94 
261 lower in patients with prostate cancer; PSP-94 is therefore a candidate biomarker for prostate 262 cancer $^{34}$. However, it is debated whether PSP-94 plays a causal role in tumorigenesis. We

263 identified a cis pQTL for PSP-94 at a prostate cancer susceptibility locus ${ }^{35}$ and showed that 264 the risk allele is associated with lower PSP-94 plasma levels, supporting a protective role for 265 the protein.

267 In an approach that extends classic MR analysis, we leveraged multi-variant MR analysis methods to distinguish causal genes among multiple plausible candidates at disease loci (Methods), exemplified by the ILIRL1-IL18RI locus. We identify four proteins that each had cis pQTLs at this locus (Supplementary Table 1), which has been associated with a range of immune-mediated diseases including atopic dermatitis ${ }^{36}$. We created a genetic score for each protein using multiple protein-increasing alleles. Initial "one-protein-at-a-time" analysis identified associations of the scores for IL18R1 $\left(p=9.3 \times 10^{-72}\right)$ and IL1RL1 $\left(p=5.7 \times 10^{-27}\right)$ with atopic dermatitis risk (Figure 5a). In addition, we found a weak association for IL1RL2 $(p=0.013)$. We then mutually adjusted these associations for one another to account for the effects of the variants on multiple proteins. While the association of IL18R1 remained significant $\left(p=1.5 \times 10^{-28}\right)$, the association of IL1RL1 $(p=0.01)$ was attenuated. In contrast, the association of IL1RL2 $\left(p=1.1 \times 10^{-69}\right)$ became much stronger, suggesting that IL1RL2 and IL18R1 are the causal proteins influencing risk of atopic dermatitis at this locus.

281 We also used multiple cis-associated variants to evaluate whether macrophage metalloelastase (MMP-12) plays a causal role in coronary disease. Since MMP-12 is known to play a role in lung damage, MMP-12 inhibitors are being tested in chronic obstructive

284 pulmonary disease (COPD). Observational studies report associations of higher levels of 285 plasma MMP-12 with recurrent cardiovascular events ${ }^{37,38}$. In contrast, our multi-allelic 
genetic score, which explains $14 \%$ of the variation in plasma MMP-12 levels (Methods),

287 indicates that genetic predisposition to higher MMP-12 levels is associated with decreased

288 coronary disease risk $\left(p=2.8 \times 10^{-13}\right)(\underline{\text { Figure } 5 \mathrm{~b}})$ and decreased large artery atherosclerotic

289 stroke risk $^{39}$. As MMP-12 is released from macrophages in response to cardiovascular

290 injury $^{40}$, it is possible that higher MMP-12 levels are cardioprotective. Hence, our results

291 identify a potential cardiovascular safety concern for MMP-12 inhibitors, particularly as patients with COPD are at high baseline cardiovascular risk due to smoking history.

\section{Drug target prioritisation}

295 Because therapeutic targets implicated by human genetic data are likely to play causal roles

296 in disease, drugs directed at such targets have greater likelihood of success ${ }^{41}$. According to

297 the Informa Pharmaprojects database (Citeline), 244 of the proteins linked to a disease-

298 associated variant by our pQTL data are drug targets (Supplementary Table 11). Of the

299 proteins we identified as associated with disease susceptibility loci, 49 are targets of already

300 approved drugs such as tocilizumab and ustekinumab (Supplementary Table 12).

301

302 To identify additional indications for existing drugs, we investigated disease associations of

303 pQTLs for proteins already targeted by licensed drugs. Our results suggest potential drug "re-

304 purposing" opportunities (Supplementary Table 12). For example, we identified a cis pQTL

305 for RANK (encoded by TNFRSF11A) at a locus associated with Paget's disease ${ }^{42}$, a condition

306 characterised by excessive bone turnover leading to deformity and fracture. Standard

307 treatment consists of osteoclast inhibition with bisphosphonates, originally developed as anti-

308 osteoporotic drugs. Denosumab, another anti-osteoporosis drug, is a monoclonal antibody

309 targeting RANKL, the ligand for RANK. Our data suggest denosumab may be a useful 
310 alternative for patients with Paget's disease in whom bisphosphonates are contra-indicated, a

311 hypothesis supported by clinical case reports ${ }^{43,44}$.

313 To evaluate targets for drugs currently under development, we considered the example of

314 glycoprotein Ib platelet alpha subunit (GP1BA), the receptor for von Willebrand factor.

315 Drugs directed at GP1BA are in pre-clinical development as anti-thrombotic agents and in

316 phase 2 trials to treat thrombotic thrombocytopenic purpura, a life-threatening disorder. We

317 identify a trans pQTL for GP1BA at the pleiotropic $S H 2 B 3 / B R A P$ locus, which is associated

318 with platelet count ${ }^{45}$, myocardial infarction and stroke. The risk allele for cardiovascular

319 disease increases both plasma GP1BA and platelet count, suggesting a mechanism by which

320 this locus affects disease susceptibility. As a confirmation of the link between GP1BA and

321 the platelet count, we found a directionally concordant cis pQTL for GP1BA at a platelet count-associated variant (Supplementary Table 12). Collectively, these results suggest that targeting GP1BA may be efficacious in conditions characterised by platelet aggregation such as arterial thrombosis. More generally, our data provide a foundation for generating hypotheses about targets for new drug development through the approach of linking genetic factors to disease via specific proteins (Supplementary Table 12).

\section{Discussion}

329 We conducted protein measurements of unprecedented scope and scale to reveal genetic

330 control of the human plasma proteome. Our discoveries enabled identification of important

331 consequences of natural perturbations in the plasma proteome. First, we identified the downstream effects of alterations in specific protein levels, revealing novel regulators of protein pathways. Second, we used plasma proteins to uncover intermediate molecular 
335 on genes in the vicinity of disease susceptibility loci. By contrast, we made key advances

336 through use of trans pQTLs to implicate previously unsuspected proteins encoded by distant

337 genes. Third, we established causal roles for protein biomarkers in vascular, neoplastic, and

338 autoimmune diseases using the principle of Mendelian randomization (MR). Proteins provide

339 an ideal paradigm for MR analysis because they are under proximal genetic control. Whereas

340 MR studies of plasma proteins have been constrained by availability of few suitable genetic

341 instruments, our data remedy this bottleneck by furnishing an extensive toolkit. Fourth, we

342 introduced an approach that should help reduce the unsustainably high attrition rates of drugs

343 in pharmaceutical pipelines. Overall, our study foreshadows major advances in post-genomic

344 science through increasing application of novel bioassay technologies to major population

345 biobanks. 


\section{Methods}

\section{Study participants}

348 The INTERVAL study comprises approximately 50,000 participants nested within a

349 randomised trial of blood donation intervals ${ }^{46}$. Between mid-2012 and mid-2014, whole-

350 blood donors aged 18 years and older were consented and recruited at 25 centers of

351 England's National Health Service Blood and Transplant. All participants completed an

352 online questionnaire including questions about demographic characteristics (e.g., age, sex,

353 ethnic group), anthropometry (height, weight), lifestyle (e.g., alcohol and tobacco

354 consumption) and diet. Participants were generally in good health because blood donation

355 criteria exclude people with a history of major disease (such as myocardial infarction, stroke,

356 cancer, HIV, and hepatitis B or C) and those who have had recent illness or infection. For

357 protein assays, we randomly selected two non-overlapping subcohorts of 2,731 and 831

358 participants from INTERVAL. Participant characteristics are shown in Supplementary Table

$359 \quad 13$.

360

\section{$361 \quad$ Plasma sample preparation}

362 Sample collection procedures for INTERVAL have been described previously ${ }^{46}$. In brief,

363 blood samples for research purposes were collected in $6 \mathrm{ml}$ EDTA tubes using standard

364 venepuncture protocols. The tubes were inverted three times and transferred at room

365 temperature to UK Biocentre (Stockport, UK) for processing. Plasma was extracted into two

$3660.8 \mathrm{ml}$ plasma aliquots by centrifugation and subsequently stored at $-80^{\circ} \mathrm{C}$ prior to use.

\section{Protein measurements}


369 We used a multiplexed, aptamer-based approach (SOMAscan assay) to measure the relative

370 concentrations of 3,620 plasma proteins/protein complexes assayed using 4,034 aptamers

371 ("SOMAmer reagents", hereafter referred to as "SOMAmers"). The assay extends the lower

372 limit of detectable protein abundance afforded by conventional approaches (e.g.,

373 immunoassays), measuring both extracellular and intracellular proteins (including soluble

374 domains of membrane-associated proteins), with a bias towards proteins likely to be found in

375 the human secretome (Extended Data Figure $7 a)^{6,47}$. The proteins cover a wide range of

376 molecular functions (Extended Data Figure 7b). The selection of proteins on the platform

377 reflects both the availability of purified protein targets and a focus on proteins known to be

378 involved in pathophysiology of human disease.

379

380 Aliquots of $150 \mu \mathrm{l}$ of plasma were sent on dry ice to SomaLogic Inc. (Boulder, Colorado, US)

381 for protein measurement. Assay details have been previously described ${ }^{47-49}$ and a technical

382 white paper with further information can be found at the manufacturer's website

383 (http://info.somalogic.com/lp_campaign_somascan-white-paper_jan-2016). In brief, modified

384 single-stranded DNA SOMAmers are used to bind to specific protein targets that are then

385 quantified using a DNA microarray. Protein concentrations are quantified as relative

386 fluorescent units.

388 Quality control (QC) was performed at the sample and SOMAmer level using control 389 aptamers, as well as calibrator samples. At the sample level, hybridization controls on the

390 microarray were used to correct for systematic variability in hybridization, while the median

391 signal over all features assigned to one of three dilution sets $(40 \%, 1 \%$ and $0.005 \%)$ was used

392 to correct for within-run technical variability. The resulting hybridization scale factors and

393 median scale factors were used to normalise data across samples within a run. The acceptance 
394 criteria for these values are between 0.4 and 2.5 based on historical runs. SOMAmer-level

395 QC made use of replicate calibrator samples using the same study matrix (plasma) to correct

396 for between-run variability. The acceptance criteria for each SOMAmer is that the calibration

397 scale factor be less than 0.4 from the median for each of the plates run. In addition, at the

398 plate level, the acceptance criteria are that the median of the calibration scale factors is

399 between 0.8 and 1.2 , while $95 \%$ of individual SOMAmers must be less than 0.4 from the 400 median within the plate.

401

402 In addition to QC processes routinely conducted by SomaLogic, we measured protein levels 403 of 30 and 10 pooled plasma samples randomly distributed across plates for subcohort 1 and 404 subcohort 2, respectively. This approach, which involved masking laboratory technicians to 405 the presence of pooled samples, enabled estimation of the reproducibility of the protein assays in directly relevant samples. We calculated coefficients of variation (CVs) for each SOMAmer within each subcohort by dividing the standard deviation by the mean of the pooled plasma sample protein readouts. In addition to passing SomaLogic QC processes, we required SOMAmers to have a $\mathrm{CV} \leqslant 20 \%$ in both subcohorts. Eight non-human protein targets were also excluded, leaving 3,283 SOMAmers (mapping to 2,994 unique proteins/protein complexes) for inclusion in the GWAS.

413 Protein mapping to UniProt identifiers and gene names was provided by SomaLogic.

414 Mapping to Ensembl gene IDs and genomic positions was performed using Ensembl Variant

415 Effects Predictor v83 (VEP) ${ }^{50}$. Protein subcellular locations were determined by exporting 416 the subcellular location annotations from UniProt ${ }^{51}$. If the term "membrane" was included in 417 the descriptor, the protein was considered to be a membrane protein, whereas if the term 418 "secreted" (but not "membrane") was included in the descriptor, the protein was considered 
419 to be a secreted protein. Proteins not annotated as either membrane or secreted proteins were 420 classified (by inference) as intracellular proteins. Proteins were mapped to molecular 421 functions using gene ontology annotations ${ }^{52}$ from UniProt.

\section{Non-genetic associations of proteins}

424 To validate the protein assays, we attempted to replicate the associations with age or sex of 42545 proteins previously reported by Ngo et al and 40 reported by Menni et al ${ }^{48,53}$. We used 426 Bonferroni-corrected $p$-value thresholds of $p=1.1 \times 10^{-3}(0.05 / 45)$ and $p=1.2 \times 10^{-3}(0.05 / 40)$ respectively. Relative protein abundances were rank-inverse normalised within each subcohort and linear regression was performed using age, sex, BMI, natural log of estimated glomerular filtration rate (eGFR) and subcohort as independent variables.

\section{Genotyping and imputation}

432 The genotyping protocol and QC for the INTERVAL samples $(n \sim 50,000)$ have been described previously in detail ${ }^{14}$. Briefly, DNA extracted from buffy coat was used to assay approximately 830,000 variants on the Affymetrix Axiom UK Biobank genotyping array at Affymetrix (Santa Clara, California, US). Genotyping was performed in multiple batches of approximately 4,800 samples each. Sample QC was performed including exclusions for sex mismatches, low call rates, duplicate samples, extreme heterozygosity and non-European descent. An additional exclusion made for this study was of one participant from each pair of close (first- or second-degree) relatives, defined as $\widehat{\pi}>0.187$. Identity-by-descent was estimated using a subset of variants with call rate $>99 \%$ and MAF $>5 \%$ in the merged dataset

441 of both subcohorts, pruned for linkage disequilibrium (LD) using PLINK v1.9 ${ }^{54}$. Numbers of 442 participants excluded at each stage of the genetic QC are summarized in Extended Data 443 Figure 1. Multi-dimensional scaling was performed using PLINK v1.9 to create components 
to account for ancestry in genetic analyses.

446 Prior to imputation, additional variant filtering steps were performed to establish a high

447 quality imputation scaffold. In summary, 654,966 high quality variants (autosomal, non448 monomorphic, bi-allelic variants with Hardy Weinberg Equilibrium (HWE) $p>5 \times 10^{-6}$, with a 449 call rate of $>99 \%$ across the INTERVAL genotyping batches in which a variant passed QC, 450 and a global call rate of $>75 \%$ across all INTERVAL genotyping batches) were used for 451 imputation. Variants were phased using SHAPEIT3 and imputed using a combined 1000 452 Genomes Phase 3-UK10K reference panel. Imputation was performed via the Sanger 453 Imputation Server (https://imputation.sanger.ac.uk) resulting in 87,696,888 imputed variants.

Prior to genetic association testing, variants were filtered in each subcohort separately using the following exclusion criteria: (1) imputation quality (INFO) score $<0.7$, (2) minor allele count $<8$, (3) HWE $p<5 \times 10^{-6}$. In the small number of cases where imputed variants had the same genomic position (GRCh37) and alleles, the variant with the lowest INFO score was removed. 10,572,788 variants passing all filters in both subcohorts were taken forward for analysis (Extended Data Figure 1).

\section{Genome-wide association study}

463 Within each subcohort, relative protein abundances were first natural log-transformed. Log 464 transformed protein levels were then adjusted in a linear regression for age, sex, duration 465 between blood draw and processing (binary, $\leq 1$ day/>1day) and the first three principal components of ancestry from multi-dimensional scaling. The protein residuals from this

467 linear regression were then rank-inverse normalized and used as phenotypes for association 468 testing. Univariate linear regression using an additive genetic model was used to test genetic 
associations. Association tests were carried out on allelic dosages to account for imputation uncertainty (“-method expected” option) using SNPTEST v2.5.2.

\section{Meta-analysis and statistical significance}

Association results from the two subcohorts were combined via fixed-effects inversevariance meta-analysis combining the betas and standard errors using METAL ${ }^{56}$. Genetic associations were considered to be genome-wide significant based on a conservative strategy requiring associations to have (i) a meta-analysis $p$-value $<1.5 \times 10^{-11}$ (genome-wide threshold of $p=5 \times 10^{-8}$ Bonferroni corrected for 3,283 aptamers tested), (ii) at least nominal significance $(p<0.05)$ in both subcohorts, and also (iii) consistent direction of effect across subcohorts. We did not observe significant genomic inflation (mean inflation factor was 1.0, standard deviation=0.01) (Extended Data Figure 8).

\section{Refinement of significant regions}

To identify distinct non-overlapping regions associated with a given SOMAmer, we first defined a $1 \mathrm{Mb}$ region around each significant variant for that SOMAmer. Starting with the region containing the variant with the smallest $p$-value, any overlapping regions were then merged and this process was repeated until no more overlapping $1 \mathrm{Mb}$ regions remained. The variant with the lowest $p$-value for each region was assigned as the "regional sentinel variant". Due to the complexity of the Major Histocompatibility Region (MHC) region, we treated the extended MHC region (chr6:25.5-34.0Mb) as one region. To identify whether a region was associated with multiple SOMAmers, we used an LD-based clumping approach.

491 Regional sentinel variants in high $\operatorname{LD}\left(r^{2} \geq 0.8\right)$ with each other were combined together into a single region. 


\section{Conditional analyses}

495 To identify conditionally significant signals, we performed approximate genome-wide step496 wise conditional analysis using GCTA v1.25.2 $2^{57}$ using the "cojo-slct" option. We used the 497 same conservative significance threshold of $p=1.5 \times 10^{-11}$ as for the univariate analysis. As 498 inputs for GCTA, we used the summary statistics (i.e. betas and standard errors) from the 499 meta-analysis. Correlation between variants was estimated using the hard-called genotypes 500 (where a genotype was called if it had a posterior probability of $>0.9$ following imputation or 501 set to missing otherwise) in the merged genetic dataset, and only variants also passing the univariate genome-wide threshold $\left(p<1.5 \times 10^{-11}\right)$ were considered for step-wise selection. As the conditional analyses use different data inputs (summarised rather than individual-level

504 data), there were some cases where the conditional analysis failed to include sentinel variants that had borderline significant univariate associations in the step-wise selection. In these instances $(n=28)$, we re-conducted the joint model estimation without step-wise selection in GCTA with variants identified by the conditional analysis in addition to the regional sentinel variant. We report and highlight these cases in Supplementary Table 2.

\section{Replication of previous pQTLs}

511 We attempted to identify all previously reported pQTLs from GWAS and to assess whether

512 they replicated in our study. We used the NCBI Entrez programming utility in R (rentrez) to 513 perform a literature search for pQTL studies published from 2008 onwards. We searched for 514 the following terms: "pQTL", "pQTLs", and "protein quantitative trait locus". We

515 supplemented this search by filtering out GWAS associations from the NHGRI-EBI GWAS 516 Catalog v.1.0.1 ${ }^{58}$ (https://www.ebi.ac.uk/gwas/, downloaded April 2016), which has all 517 phenotypes mapped to the Experimental Factor Ontology (EFO) ${ }^{59}$, by restricting to those 518 with EFO annotations relevant to protein biomarkers (e.g. "protein measurement", 
519 EFO_0004747). Studies identified through both approaches were manually filtered to include only studies that profiled plasma or serum samples and to exclude studies not assessing

521 proteins. We recorded basic summary information for each study including the assay used, 522 sample size and number of proteins with pQTLs (Supplementary Table 14). To reduce the 523 impact of ethnic differences in allele frequencies on replication rate estimates, we filtered 524 studies to include only associations reported in European-ancestry populations. We then manually extracted summary data on all reported associations from the manuscript or the supplementary material. This included rsID, protein UniProt ID, $p$-values, and whether the association is cis/trans (Supplementary Table 15).

529 To assess replication we first identified the set of unique UniProt IDs that were also assayed on the SOMAscan panel. For previous studies that used SomaLogic technology, we refined this match to the specific aptamer used. We then clumped associations into distinct loci using the same method applied to our pQTL (see Refinement of significant regions). For each

533 locus, we asked if the sentinel SNP or a proxy $\left(r^{2}>0.6\right)$ was associated with the same 534 protein/aptamer in our study at a defined significance threshold. For our primary assessment, we used a $p$-value threshold of $10^{-4}$. We also performed sensitivity analyses to explore factors that influence replication rate (Supplementary Note).

\section{Candidate gene annotation}

539 We defined a pQTL as cis when the most significantly associated variant in the region was

540 located within $1 \mathrm{Mb}$ of the transcription start site (TSS) of the gene(s) encoding the protein.

541 pQTLs lying outside of the region were defined as trans. When considering the distance of 542 the top cis-associated variant from the relevant TSS, only proteins that map to single genes 543 on the primary assembly in Ensembl v83 were considered. 
545 For trans pQTLs, we sought to prioritise candidate genes in the region that might underpin

546 the genotype-protein association. In addition to reporting the nearest gene to the sentinel

547 variant, we employed "bottom up" and "top down" approaches, starting from the variant and

548 protein respectively For the "bottom up" approach, the sentinel variant and corresponding

549 proxies $\left(r^{2}>0.8\right)$ for each trans pQTL were first annotated using Ensembl VEP v83 (using the

550 "pick" option) to determine whether variants were (1) protein-altering coding variants; (2)

551 synonymous coding or 5'/3' untranslated region (UTR); (3) intronic or up/downstream; or (4)

552 intergenic. Second, we queried all sentinel variants and proxies against significant cis eQTL

553 variants (defined by beta distribution-adjusted empirical $p$-values using a FDR threshold of

5540.05 , see http://www.gtexportal.org/home/documentationPage for details) in any cell type or

555 tissue from the Genotype-Tissue Expression (GTEx) project $6^{60}$

556 (http://www.gtexportal.org/home/datasets). Third, we also queried promoter capture Hi-C

557 data in 17 human primary hematopoietic cell types ${ }^{61}$ to identify contacts (with a CHICAGO

558 score $>5$ in at least one cell type) involving chromosomal regions containing a sentinel

559 variant. We considered gene promoters annotated on either fragment (i.e., the fragment

560 containing the sentinel variant or the other corresponding fragment) as potential candidate

561 genes. Using these three sources of information, we generated a list of candidate genes for the

562 trans pQTLs. A gene was considered a candidate if it fulfilled at least one of the following

563 criteria: (1) it was proximal (intragenic or $\pm 5 \mathrm{~Kb}$ from the gene) or nearest to the sentinel

564 variant; (2) it contained a sentinel or proxy variant $\left(r^{2}>0.8\right)$ that was protein-altering; (3) it

565 had a significant cis eQTL in at least one GTEx tissue overlapping with a sentinel pQTL

566 variant (or proxy); or (4) it was regulated by a promoter annotated on either fragment of a

567 chromosomal contact ${ }^{61}$ involving a sentinel variant. 
569 For the "top down" approach, we first identified all genes with a TSS located within the

570 corresponding pQTL region using the GenomicRanges Bioconductor package ${ }^{62}$ with

571 annotation from a GRCh37 GTF file from

572 (ftp://ftp.ensembl.org/pub/grch37/update/gtf/homo sapiens/; file:

573 "Homo_sapiens.GRCh37.82.gtf.gz", downloaded June 2016). We then identified any local

574 genes that had previously been linked with the corresponding trans-associated protein(s)

575 according to the following open source databases: (1) the Online Mendelian Inheritance in

576 Man (OMIM) catalogue ${ }^{63}$ (http://www.omim.org/); (2) the Kyoto Encyclopedia of Genes and

577 Genomes (KEGG) ${ }^{64}$ (http://www.genome.jp/kegg/); and (3) STRINGdb ${ }^{65}$ (http://string-

578 db.org/; v10.0). We accessed OMIM data via HumanMine web tool ${ }^{66}$

579 (http://www.humanmine.org/; accessed June 2016), whereby we extracted all OMIM IDs for

580 (i) our trans-affected proteins and (ii) genes local $( \pm 500 \mathrm{~Kb})$ to the corresponding trans-acting

581 variant. We extracted all human KEGG pathway IDs using the KEGGREST Bioconductor

582 package $^{67}$ (https://bioconductor.org/packages/release/bioc/html/KEGGREST.html). In cases

583 where a trans-associated protein shared either an OMIM ID or a KEGG pathway ID with a

584 gene local to the corresponding trans-acting variant, we took this as evidence of a potential

585 functional involvement of that gene. We interrogated protein-protein interaction data by

586 accessing STRINGdb data using the STRINGdb Bioconductor package ${ }^{68}$, whereby we

587 extracted all pairwise interaction scores for each trans-affected protein and all proteins with

588 genes local to the corresponding trans-acting variants. We took the default interaction score

589 of 400 as evidence of an interaction between the proteins, therefore indicating a possible

590 functional involvement for the local gene. In addition to using data from open source

591 databases in our top-down approach we also adopted a "guilt-by-association" (GbA)

592 approach utilising the same plasma proteomic data used to identify our pQTLs. We first

593 generated a matrix containing all possible pairwise Pearson's correlation coefficients between 
our 3,283 SOMAmers. We then extracted the coefficients relating to our trans-associated proteins and any proteins encoded by genes local to their corresponding trans-acting variants (where available). Where the correlation coefficient was $\geqslant 0.5$ we prioritised the relevant local genes as being potential mediators of the trans-signal(s) at that locus.

We report the potential candidate genes for our trans pQTLs from both the "bottom up" and 600 "top down" approaches, highlighting cases where the same gene was highlighted by both approaches.

602

\section{Functional annotation of pQTLs}

604 Functional annotation of variants was performed using Ensembl VEP v83 using the "pick" 605 option. We tested the enrichment of significant pQTL variants for certain functional classes 606 by comparing to permuted sets of variants showing no significant association with any 607 protein $(p>0.0001$ for all proteins tested). First the regional sentinel variants were LD-pruned 608 at $r^{2}$ of 0.1 . Each time the sentinel variants were LD-pruned, one of the pair of correlated 609 variants was removed at random and for each set of LD-pruned sentinel variants and 100 sets 610 of equally sized null permuted variants were sampled matching for MAF (bins of 5\%), 611 distance to TSS (bins of $0-0.5 \mathrm{~Kb}, 0.5 \mathrm{~Kb}-2 \mathrm{~Kb}, 2 \mathrm{~Kb}-5 \mathrm{~Kb}, 10 \mathrm{~Kb}-20 \mathrm{~Kb}, 20 \mathrm{~Kb}-100 \mathrm{~Kb}$ and $612>100 \mathrm{~Kb}$ in each direction) and $\mathrm{LD}( \pm$ half the number of variants in LD with the sentinel 613 variant at $r^{2}$ of 0.8 ). This procedure was repeated 100 times resulting in 10,000 permuted sets 614 of variants. An empirical $p$-value was calculated as the proportion of permuted variant sets 615 where the proportion that are classified as a particular functional group exceeded that of the 616 test set of sentinel pQTL variants, and we used a significance threshold of $p=0.005(0.05 / 10$ 617 functional classes tested). 


\section{Evidence against aptamer-binding effects at cis pQTLs}

620 All protein assays that rely on binding (e.g. of antibodies or SOMAmers) are susceptible to 621 the possibility of binding-affinity effects, where protein-altering variants (PAVs) (or their 622 proxies in LD) are associated with protein measurements due to differential binding rather 623 than differences in protein abundance. To account for this potential effect, we performed 624 conditional analysis at all cis pQTLs where the sentinel variant was in $\operatorname{LD}\left(0.1 \leq r^{2} \leqslant 0.9\right)$ with 625 a PAV in the gene(s) encoding the associated protein. First, variants were annotated with 626 Ensemb1 VEP v83 using the "per-gene" option. Variant annotations were considered protein 627 altering if they were annotated as coding sequence variant, frameshift variant, in-frame 628 deletion, in-frame insertion, missense variant, protein altering variant, splice acceptor variant, splice donor variant, splice region variant, start lost, stop gained, or stop lost. To avoid multi630 collinearity, PAVs were LD-pruned $\left(r^{2}>0.9\right)$ using PLINK v1.9 before including them as 631 covariates in the conditional analysis on the meta-analysis summary statistics using GCTA v1.25.2. Any variants with an $r^{2} \geq 0.9$ with any of the PAVs were removed. Coverage of known common (MAF>5\%) PAVs in our data was checked by comparison with exome

634 sequences from $\sim 60,000$ individuals in the Exome Aggregation Consortium ${ }^{69}$ (ExAC 635 [http://exac.broadinstitute.org], downloaded June 2016).

636

\section{Testing for regulatory and functional enrichment}

638 We tested whether our pQTLs were enriched for functional and regulatory characteristics using GARFIELD v1.2.0 ${ }^{18}$. GARFIELD is a non-parametric permutation-based enrichment

640 method that compares input variants to permuted sets matched for number of proxies $\left(r^{2} \geqslant\right.$

641 0.8), MAF and distance to the closest TSS. It first applies "greedy pruning" $\left(r^{2}<0.1\right)$ within a $6421 \mathrm{Mb}$ region of the most significant variant. GARFIELD annotates variants with more than a 643 thousand features, drawn predominantly from the GENCODE, ENCODE and ROADMAP 
644 projects, which includes genic annotations, histone modifications, chromatin states and other

645 regulatory features across a wide range of tissues and cell types.

646

647 The enrichment analysis was run using all variants that passed our Bonferroni-adjusted

648 significance threshold $\left(p<1.5 \times 10^{-11}\right)$ for association with any protein. For each of the 649 matching criteria (MAF, distance to TSS, number of LD proxies), we used five bins. In total 650 we tested 25 combinations of features (classified as transcription factor binding sites, FAIRE651 seq, chromatin states, histone modifications, footprints, hotspots, or peaks) with up to 190 652 cell types from 57 tissues, leading to 998 tests. Hence, we considered enrichment with a $p<5$ $653 \times 10^{-5}(0.05 / 998)$ to be statistically significant.

\section{Disease annotation}

656 To identify diseases that our pQTLs have been associated with, we queried our sentinel 657 variants and their strong proxies $\left(r^{2} \geq 0.8\right)$ against publicly available disease GWAS data using 658 PhenoScanner $^{8}$ A list of datasets queried is available at 659 http://www.phenoscanner.medschl.cam.ac.uk/information.html. For disease GWAS, results were filtered to $p<5 \times 10^{-8}$ and then manually curated to retain only the entry with the strongest evidence for association (i.e. smallest $p$-value) per disease. Non-disease phenotypes such as anthropometric traits, intermediate biomarkers and lipids were excluded manually.

\section{Cis eQTL overlap and enrichment of cis pQTLs for cis eQTLs}

665 Each regional sentinel cis variant its strong proxies $\left(r^{2} \geq 0.8\right)$ were queried against publicly available eQTL association data using PhenoScanner. Cis eQTL results were filtered to retain

667 only variants with $p<1.5 \times 10^{-11}$. Only cis eQTLs for the same gene as the $c$ is pQTL protein were retained. To assess whether our cis pQTLs were more likely to also be cis eQTLs than 
non-pQTL variants, we used data from the GTEx project v6, due to the availability of genome-wide association results across a wide range of tissues and cell-types. GTEx results were filtered to contain only variants lying in cis (i.e., within $1 \mathrm{Mb}$ ) of genes that encode proteins analysed in our study and only variants in both datasets were utilised.

674 For the enrichment analysis, the cis pQTL sentinel variants were first LD-pruned $\left(r^{2}<0.1\right)$ and the proportion of sentinel cis pQTL variants that are also eQTLs (at $\left.p<1.5 \times 10^{-11}\right)$ for the same protein/gene was compared to a permuted set of variants that were not pQTLs ( $p>0.0001$ for all proteins). We generated 10,000 permuted sets of null variants matched for MAF, distance to TSS and LD (as described for functional annotation enrichment in

Functional annotation of pQTLs). An empirical $p$-value was calculated as the proportion of permuted variant sets where the proportion that are also cis eQTLs exceeded that of the test set of sentinel cis pQTL variants. Results were similar in sensitivity analyses using the standard genome-wide significance threshold of $p<5 \times 10^{-8}$ for the eQTLs $(13.0 \%$ for whole blood, $18.7 \%$ for LCLs, $17.1 \%$ for liver and $13.4 \%$ for monocytes) as well as also using only the sentinel variants at cis pQTLs that were robust to adjusting for PAVs, suggesting our results are robust to choice of threshold and potential differential binding effects.

686

\section{Mediation of the GDF11/8 trans pQTL by WFIKKN2 levels}

To assess whether the trans pQTL for GDF11/8 in the WFIKKN2 gene region was mediated by WFIKKN2 levels, we first regressed the rank inverse transformed residuals for GDF11/8 used for the GWAS against the WFIKKN2 residuals, adjusting for subcohort. The residuals

691 from this regression were subsequently regressed against allelic dosages for each variant in the WFIKKN2 region. As there were two SOMAmers targeting WFIKKN2, we tested both to see if similar results were obtained. Regional association plots were made using Gviz ${ }^{70}$. 


\section{Selection of genetic instruments for Mendelian randomization}

696 In Mendelian randomization (MR), genetic variants are used as "instrumental variables" (IV)

697 for assessing the causal effect of the exposure (here a plasma protein) on the outcome (here 698 disease).

699

\section{$700 \quad$ Proteins in the $I L 1 R L 1-I L 18 R 1$ locus and atopic dermatitis}

701 To identify the likely causal proteins that underpin the previous genetic association of the IL1RL1-IL18R1 locus (chr11:102.5-103.5Mb) with atopic dermatitis (AD) ${ }^{36}$, we used a multivariable MR approach. For each protein encoded by a gene in the ILIRL1-ILI8R1 locus, we took genetic variants that had a cis association at $p<1 \times 10^{-4}$ and 'LD-pruned' them at $r^{2}<0.1$. We then used these variants as instrumental variables for their respective proteins in univariate MR. For multivariable MR, association estimates for all proteins in the locus were extracted for all instruments. We used PhenoScanner to obtain association statistics for the selected variants in the European-ancestry population of a recent large-scale GWAS metaanalysis $^{36}$. Where the relevant variant was not available, the strongest proxy with $r^{2} \geqslant 0.8$ was used.

MMP-12 and coronary heart disease (CHD)

To test whether plasma MMP-12 levels have a causal effect on risk of CHD, we selected

714 genetic variants in the MMP12 gene region to use as instrumental variables. We constructed a genetic score comprising 17 variants that had a cis association with MMP-12 levels at

$716 p<5 \times 10^{-8}$ and that were not highly correlated with one another $\left(r^{2}<0.2\right)$. To perform 717 multivariable MR, we used association estimates for these variants with other MMP proteins 718 in the locus (MMP-1, MMP-7, MMP-8, MMP-10, MMP-13). Summary associations for 
719 variants in the score with CHD were obtained through PhenoScanner from a recent largescale 1000 Genomes-based GWAS meta-analysis which consists mostly (77\%) of individuals of European ancestry ${ }^{71}$.

722

\section{MR analysis}

724 Two-sample univariate MR was performed for each protein separately using summary 725 statistics in the inverse-variance weighted method adapted to account for correlated 726 variants $^{72,73}$. For each of $G$ genetic variants $(g=1, \ldots, G)$ having per-allele estimate of the association with the protein $\beta_{X g}$ and standard error $\sigma_{X g}$, and per-allele estimate of the association with the outcome (here, $\mathrm{AD}$ or $\mathrm{CHD}$ ) $\beta_{Y g}$ and standard error $\sigma_{Y g}$, the IV estimate $\left(\hat{\theta}_{X Y}\right)$ is obtained from generalised weighted linear regression of the genetic associations with

730 the outcome $\left(\beta_{Y}\right)$ on the genetic associations with the protein $\left(\beta_{X}\right)$ weighting for the precisions of the genetic associations with the outcome and accounting for correlations between the variants according to the regression model:

$$
\beta_{Y}=\theta_{X Y} \beta_{X}+\varepsilon, \quad \varepsilon \sim N(0, \Omega)
$$

where $\beta_{Y}$ and $\beta_{X}$ are vectors of the univariable (marginal) genetic associations, and the weighting matrix $\Omega$ has terms $\Omega_{g_{1} g_{2}}=\sigma_{Y g_{1}} \sigma_{Y g_{2}} \rho_{g_{1} g_{2}}$, and $\rho_{g_{1} g_{2}}$ is the correlation between the $g_{1}$ th and $g_{2}$ th variants.

$$
\hat{\theta}_{X Y}=\left(\beta_{X}{ }^{T} \Omega^{-1} \beta_{X}\right)^{-1} \beta_{X}{ }^{T} \Omega^{-1} \beta_{Y}
$$


and the standard error is:

$$
\operatorname{se}\left(\hat{\theta}_{X Y}\right)=\sqrt{\left(\beta_{X}{ }^{T} \Omega^{-1} \beta_{X}\right)^{-1}}
$$

748 where ${ }^{T}$ is a matrix transpose. This is the estimate and standard error from the regression analysis).

751

Genetic variants in univariate MR need to satisfy three key assumptions to be valid instruments:

(1) the variant is associated with the risk factor of interest (i.e., the protein level),

760 To account for potential effects of functional pleiotropy ${ }^{74}$, we performed multivariable MR

761 using the weighted regression-based method proposed by Burgess $e a^{75}$. For each of $K$ risk

762 factors in the model $(k=1, \ldots, K)$, the weighted regression-based method is performed by multivariable generalized weighted linear regression of the association estimates $\beta_{Y}$ on each of the association estimates with each risk factor $\beta_{X k}$ in a single regression model:

$$
\beta_{Y}=\theta_{X Y 1} \beta_{X 1}+\theta_{X Y 2} \beta_{X 2}+\cdots+\theta_{X Y K} \beta_{X K}+\varepsilon, \quad \varepsilon \sim N(0, \Omega)
$$


where $\beta_{X 1}$ is the vectors of the univariable genetic associations with risk factor 1 , and so on.

769 This regression model is implemented by first pre-multiplying the association vectors by the

770 Cholesky decomposition of the weighting matrix, and then applying standard linear

771 regression to the transformed vectors. Estimates and standard errors are obtained fixing the

772 residual standard error to be 1 as above.

773

774 The multivariable MR analysis allows the estimation of the causal effect of a protein on disease outcome accounting for the fact that genetic variants may be associated with multiple proteins in the region. Causal estimates from multivariable MR represent direct causal effects, representing the effect of intervening on one risk factor in the model while keeping others constant.

779

\section{MMP-12 genetic score sensitivity analyses}

We performed two sensitivity analyses to determine the robustness of the Mendelian randomization findings. First, we measured plasma MMP-12 levels using a different method

783 (proximity extension assay; Olink Bioscience, Uppsala, Sweden ${ }^{76,77}$ ) in a sub-sample of 141 individuals, and used this to derive genotype-MMP12 effect estimates for the 17 variants in our genetic score. Second, we obtained effect estimates from a pQTL study based on SOMAscan assay measurements in an independent sample of $\sim 1,000$ individuals ${ }^{7}$. In both cases the genetic score reflecting higher plasma MMP-12 was associated with lower risk of CHD.

\section{Overlap of pQTLs with drug targets}

791 We used the Informa Pharmaprojects database from Citeline to obtain information on drugs 792 that target proteins assayed on the SOMAscan platform. This is a manually curated database 
793 that maintains profiles for $>60,000$ drugs. For our analysis, we focused on the following

794 information for each drug: protein target, indications, and development status. We included

795 drugs across the development pipeline, including those in pre-clinical studies or with no

796 development reported, drugs in clinical trials (all phases), and launched/registered drugs. For

797 each protein assayed, we identified all drugs in the Informa Pharmaprojects with a matching

798 protein target based on UniProt ID. When multiple drugs targeted the same protein, we

799 selected the drug with the latest stage of development.

800

801 For drug targets with significant pQTLs, we identified the subset where the sentinel variant or

802 proxy variants in LD $\left(r^{2}>0.6\right)$ are also associated with disease risk through PhenoScanner.

803 We used an internal Merck auto-encoding method to map GWAS traits and drug indications

804 to a common set of terms from the Medical Dictionary for Regulatory Activities (MedDRA).

805 MedDRA terms are organized into a hierarchy with five levels. We mapped each GWAS trait

806 and indication onto the 'Lowest Level Terms' (i.e. the most specific terms available). All

807 matching terms were recorded for each trait or indication. We matched GWAS traits to drug

808 indications based on the highest level of the hierarchy, called 'System Organ Class' (SOC).

809 We designated a protein as 'matching' if at least one GWAS trait term matched with at least

810 one indication term for at least one drug.

811

\section{Data availability}

813 Summary association results will be made available on publication through

814 http://www.phenoscanner.medschl.cam.ac.uk. 


\section{References}

816 1. Albert, F. W. \& Kruglyak, L. The role of regulatory variation in complex traits and disease. Nat. Rev. Genet. 16, 197-212 (2015).

819

820

2. Liu, Y. et al. Quantitative variability of 342 plasma proteins in a human twin population. Mol. Syst. Biol. 11, 786 (2015).

821

822

823

824

825

826

827

828

829

830

831

832

833

834

835

836

837

838

839

840

841

842

843

844

845

846

847

848

3. Melzer, D. et al. A Genome-Wide Association Study Identifies Protein Quantitative Trait Loci (pQTLs). PLoS Genet 4, e1000072 (2008).

4. Enroth, S., Johansson, Å., Enroth, S. B. \& Gyllensten, U. Strong effects of genetic and lifestyle factors on biomarker variation and use of personalized cutoffs. Nat Commun 5, (2014).

5. Deming, Y. et al. Genetic studies of plasma analytes identify novel potential biomarkers for several complex traits. Sci. Rep. 6, 18092 (2016).

6. Rohloff, J. C. et al. Nucleic Acid Ligands With Protein-like Side Chains: Modified Aptamers and Their Use as Diagnostic and Therapeutic Agents. Mol. Ther. - Nucleic Acids 3, e201 (2014).

7. Suhre, K. et al. Connecting genetic risk to disease end points through the human blood plasma proteome. Nat. Commun. 8, 14357 (2017).

8. Staley, J. R. et al. PhenoScanner: a database of human genotype-phenotype associations. Bioinformatics 32, 3207-3209 (2016).

9. Burgess, S. et al. Using published data in Mendelian randomization: a blueprint for efficient identification of causal risk factors. Eur. J. Epidemiol. 30, 543-52 (2015).

10. Stranger, B. E. et al. Patterns of cis regulatory variation in diverse human populations. PLoS Genet. 8, e1002639 (2012).

11. Montgomery, S. B. \& Dermitzakis, E. T. From expression QTLs to personalized transcriptomics. Nat. Rev. Genet. 12, 277-282 (2011).

12. Battle, A. et al. Characterizing the genetic basis of transcriptome diversity through RNA-sequencing of 922 individuals. Genome Res. 24, 14-24 (2014).

13. Walter, K. et al. The UK10K project identifies rare variants in health and disease. Nature 526, 82-90 (2015).

14. Astle, W. J. et al. The Allelic Landscape of Human Blood Cell Trait Variation and Links to Common Complex Disease. Cell 167, 1415-1429.e19 (2016).

15. Harrow, J. et al. GENCODE: the reference human genome annotation for The ENCODE Project. Genome Res. 22, 1760-74 (2012).

16. Dunham, I. et al. An integrated encyclopedia of DNA elements in the human genome. Nature 489, 57-74 (2012).

850 17. Bernstein, B. E. et al. The NIH Roadmap Epigenomics Mapping Consortium. Nat. Biotechnol. 28, 1045-8 (2010).

18. Iotchkova, V. et al. GARFIELD - GWAS Analysis of Regulatory or Functional Information Enrichment with LD correction. bioRxiv (2016). at http://biorxiv.org/content/early/2016/11/07/085738.abstract

19. $\mathrm{Wu}, \mathrm{L}$. et al. Variation and genetic control of protein abundance in humans. Nature 499, 79-82 (2013).

20. Westra, H.-J. et al. Systematic identification of trans eQTLs as putative drivers of known disease associations. Nat Genet 45, 1238-1243 (2013).

21. Lappalainen, T. et al. Transcriptome and genome sequencing uncovers functional variation in humans. Nature 501, 506-511 (2013).

861 22. Schadt, E. E. et al. Mapping the Genetic Architecture of Gene Expression in Human 862 Liver. PLoS Biol. 6, e107 (2008). 
23. Zeller, T. et al. Genetics and Beyond - The Transcriptome of Human Monocytes and Disease Susceptibility. PLoS One 5, e10693 (2010).

24. Nai, A. et al. TMPRSS6 rs855791 modulates hepcidin transcription in vitro and serum hepcidin levels in normal individuals. Blood 118, (2011).

25. Carrasquillo, M. M. et al. Genome-wide Screen Identifies rs646776 near Sortilin as a Regulator of Progranulin Levels in Human Plasma. Am. J. Hum. Genet. 87, 890-897 (2010).

26. Schuelke, M. et al. Myostatin Mutation Associated with Gross Muscle Hypertrophy in a Child. N. Engl. J. Med. 350, 2682-2688 (2004).

27. Malik, V., Rodino-Klapac, L. R. \& Mendell, J. R. Emerging drugs for Duchenne muscular dystrophy. Expert Opin. Emerg. Drugs 17, 261-277 (2012).

28. Kondás, K., Szláma, G., Trexler, M. \& Patthy, L. Both WFIKKN1 and WFIKKN2 have high affinity for growth and differentiation factors 8 and 11. J. Biol. Chem. 283, 23677-84 (2008).

29. Jostins, L. et al. Host-microbe interactions have shaped the genetic architecture of inflammatory bowel disease. Nature 491, 119-124 (2012).

30. Liu, J. Z. et al. Association analyses identify 38 susceptibility loci for inflammatory bowel disease and highlight shared genetic risk across populations. Nat. Genet. 47, 979-986 (2015).

31. Di Narzo, A. F. et al. High-Throughput Characterization of Blood Serum Proteomics of IBD Patients with Respect to Aging and Genetic Factors. PLOS Genet. 13, e1006565 (2017).

32. Lyons, P. A. et al. Genetically Distinct Subsets within ANCA-Associated Vasculitis. N. Engl. J. Med. 367, 214-223 (2012).

33. Hingorani, A. \& Humphries, S. Nature's randomised trials. Lancet 366, 1906-8 (2005).

34. Grönberg, H. et al. Prostate cancer screening in men aged 50-69 years (STHLM3): a prospective population-based diagnostic study. Lancet Oncol. 16, 1667-1676 (2015).

35. Eeles, R. A. et al. Multiple newly identified loci associated with prostate cancer susceptibility. Nat. Genet. 40, 316-321 (2008).

36. Paternoster, L. et al. Multi-ancestry genome-wide association study of 21,000 cases and 95,000 controls identifies new risk loci for atopic dermatitis. Nat. Genet. 47, 1449-56 (2015).

37. Ganz, P. et al. Development and Validation of a Protein-Based Risk Score for Cardiovascular Outcomes Among Patients With Stable Coronary Heart Disease. JAMA 315, 2532 (2016).

38. Goncalves, I. et al. Elevated Plasma Levels of MMP-12 Are Associated With Atherosclerotic Burden and Symptomatic Cardiovascular Disease in Subjects With Type 2 DiabetesSignificance. Arterioscler. Thromb. Vasc. Biol. 35, 1723-1731 (2015).

39. Traylor, M. et al. A Novel MMP12 Locus Is Associated with Large Artery Atherosclerotic Stroke Using a Genome-Wide Age-at-Onset Informed Approach. PLoS Genet. 10, e1004469 (2014).

40. Pinto, A. R., Godwin, J. W. \& Rosenthal, N. A. Macrophages in cardiac homeostasis, injury responses and progenitor cell mobilisation. Stem Cell Res. 13, 705-714 (2014).

41. Nelson, M. R. et al. The support of human genetic evidence for approved drug indications. Nat. Genet. 47, 856-860 (2015).

42. Albagha, O. M. E. et al. Genome-wide association study identifies variants at CSF1, OPTN and TNFRSF11A as genetic risk factors for Paget's disease of bone. Nat. Genet. 42, 520-524 (2010).

43. Schwarz, P., Rasmussen, A. Q., Kvist, T. M., Andersen, U. B. \& Jørgensen, N. R. 
Paget's disease of the bone after treatment with Denosumab: a case report. Bone 50, 1023-5 (2012).

44. Polyzos, S. A. et al. Denosumab Treatment for Juvenile Paget's Disease: Results From Two Adult Patients With Osteoprotegerin Deficiency ('Balkan' Mutation in the TNFRSF11B Gene). J. Clin. Endocrinol. Metab. 99, 703-707 (2014).

45. Gieger, C. et al. New gene functions in megakaryopoiesis and platelet formation. Nature 480, 201-208 (2011).

46. Moore, C. et al. The INTERVAL trial to determine whether intervals between blood donations can be safely and acceptably decreased to optimise blood supply: study protocol for a randomised controlled trial. Trials 15, 363 (2014).

47. Gold, L. et al. Aptamer-Based Multiplexed Proteomic Technology for Biomarker Discovery. PLoS One 5, e15004 (2010).

48. Menni, C. et al. Circulating Proteomic Signatures of Chronological Age. J Gerontol A Biol Sci Med Sci (2014). doi:10.1093/gerona/glu121

49. Sattlecker, M. et al. Alzheimer's disease biomarker discovery using SOMAscan multiplexed protein technology. Alzheimer's Dement. 10, 724-734 (2014).

50. McLaren, W. et al. Deriving the consequences of genomic variants with the Ensembl API and SNP Effect Predictor. Bioinformatics 26, 2069-70 (2010).

51. UniProt Consortium. UniProt: a hub for protein information. Nucleic Acids Res. 43, D204-D212 (2015).

52. Ashburner, M. et al. Gene Ontology: tool for the unification of biology. Nat. Genet. 25, 25-29 (2000).

53. Ngo, D. et al. Aptamer-Based Proteomic Profiling Reveals Novel Candidate Biomarkers and Pathways in Cardiovascular Disease. Circulation 134, (2016).

54. Chang, C. C. et al. Second-generation PLINK: rising to the challenge of larger and richer datasets. Gigascience 4, 7 (2015).

55. Marchini, J., Howie, B., Myers, S., McVean, G. \& Donnelly, P. A new multipoint method for genome-wide association studies by imputation of genotypes. Nat. Genet. 39, 906-913 (2007).

56. Willer, C. J., Li, Y. \& Abecasis, G. R. METAL: fast and efficient meta-analysis of genomewide association scans. Bioinformatics 26, 2190-1 (2010).

57. Yang, J. et al. Conditional and joint multiple-SNP analysis of GWAS summary statistics identifies additional variants influencing complex traits. Nat. Genet. 44, 36975, S1-3 (2012).

58. Welter, D. et al. The NHGRI GWAS Catalog, a curated resource of SNP-trait associations. Nucleic Acids Res. 42, D1001-6 (2014).

59. Malone, J. et al. Modeling sample variables with an Experimental Factor Ontology. Bioinformatics 26, 1112-1118 (2010).

60. Aguet, F. et al. Local genetic effects on gene expression across 44 human tissues. bioRxiv (2016). at http://biorxiv.org/content/early/2016/09/09/074450.abstract

61. Javierre, B. M. et al. Lineage-Specific Genome Architecture Links Enhancers and Non-coding Disease Variants to Target Gene Promoters. Cell 167, 1369-1384.e19 (2016).

62. Lawrence, M. et al. Software for Computing and Annotating Genomic Ranges. PLoS Comput. Biol. 9, e1003118 (2013).

63. Amberger, J. S., Bocchini, C. A., Schiettecatte, F., Scott, A. F. \& Hamosh, A. OMIM.org: Online Mendelian Inheritance in Man (OMIM(R)), an online catalog of human genes and genetic disorders. Nucleic Acids Res. 43, D789-D798 (2015).

64. Kanehisa, M., Sato, Y., Kawashima, M., Furumichi, M. \& Tanabe, M. KEGG as a reference resource for gene and protein annotation. Nucleic Acids Res. 44, D457-D462 
(2016).

65. Szklarczyk, D. et al. STRING v10: protein-protein interaction networks, integrated over the tree of life. Nucleic Acids Res. 43, D447-D452 (2015).

66. Smith, R. N. et al. InterMine: a flexible data warehouse system for the integration and analysis of heterogeneous biological data. Bioinformatics 28, 3163-3165 (2012).

67. Tenenbaum, D. KEGGREST: Client-side REST access to KEGG. (2016).

68. Franceschini, A. et al. STRING v9.1: protein-protein interaction networks, with increased coverage and integration. Nucleic Acids Res. 41, D808-D815 (2013).

69. Lek, M. et al. Analysis of protein-coding genetic variation in 60,706 humans. Nature 536, 285-291 (2016).

70. Hahne, F. \& Ivanek, R. in Methods in molecular biology (Clifton, N.J.) 1418, 335-351 (2016).

71. Nikpay, M. et al. A comprehensive 1,000 Genomes-based genome-wide association meta-analysis of coronary artery disease. Nat. Genet. 47, 1121-30 (2015).

72. Burgess, S., Butterworth, A. \& Thompson, S. G. Mendelian randomization analysis with multiple genetic variants using summarized data. Genet. Epidemiol. 37, 658-665 (2013).

73. Burgess, S., Dudbridge, F. \& Thompson, S. G. Combining information on multiple instrumental variables in Mendelian randomization: comparison of allele score and summarized data methods. Stat. Med. 35, 1880-906 (2016).

74. Burgess, S. \& Thompson, S. G. Multivariable Mendelian randomization: the use of pleiotropic genetic variants to estimate causal effects. Am. J. Epidemiol. 181, 251-60 (2015).

75. Burgess, S., Dudbridge, F. \& Thompson, S. G. Re: 'Multivariable Mendelian randomization: the use of pleiotropic genetic variants to estimate causal effects'. Am. J. Epidemiol. 181, 290-1 (2015).

76. Lundberg, M., Eriksson, A., Tran, B., Assarsson, E. \& Fredriksson, S. Homogeneous antibody-based proximity extension assays provide sensitive and specific detection of low-abundant proteins in human blood. Nucleic Acids Res. 39, e102-e102 (2011).

77. Assarsson, E. et al. Homogenous 96-plex PEA immunoassay exhibiting high sensitivity, specificity, and excellent scalability. PLoS One 9, e95192 (2014). 


\section{Supplementary Information}

996 Supplementary Information is available in the online version of the paper.

\section{Acknowledgements}

998 We acknowledge the participation of all INTERVAL volunteers. We thank the INTERVAL 999 study co-ordination teams (at the Universities of Cambridge and Oxford and at NHS Blood 1000 and Transplant [NHSBT]), including the blood donation staff at the 25 static centers, for their 1001 help with INTERVAL participant recruitment and study fieldwork, as well as the Cambridge 1002 BioResource and NHSBT staff for their help with volunteer recruitment. We thank the 1003 INTERVAL Operations Team headed by Dr Richard Houghton and Dr Carmel Moore, and 1004 the INTERVAL Data Management Team headed by Dr Matthew Walker. We thank all the 1005 staff at SomaLogic for processing and running the proteomic assays. We thank Aaron Day1006 Williams, Joshua McElwee, Dorothee Diogo, William Astle, Emanuele Di Angelantonio, 1007 Ewan Birney, Arianne Richard, Justin Mason and Michael Inouye for helpful comments on 1008 the manuscript and Mark Sharp for help mapping drug indications to GWAS traits. The $1009 \mathrm{MRC} / \mathrm{BHF}$ Cardiovascular Epidemiology Unit is supported by the UK Medical Research 1010 Council (G0800270), British Heart Foundation (SP/09/002), UK National Institute for Health

1011 Research Cambridge Biomedical Research Centre, European Research Council (268834), and 1012 European Commission Framework Programme 7 (HEALTH-F2-2012-279233). B.B.S. is

1013 funded by the Cambridge School of Clinical Medicine MRC/Sackler Prize PhD Studentship $1014(\mathrm{MR} / \mathrm{K} 50127 \mathrm{X} / 1)$ and supported by the Cambridge School of Clinical Medicine MB-PhD 1015 programme. J.E.P. is funded by a British Heart Foundation Clinical Research Fellowship 1016 through the BHF Cambridge Centre of Excellence [RE/13/6/30180]. D.S.P. and D.S. are 1017 funded by the Wellcome Trust (105602/Z/14/Z). N.S. is supported by the Wellcome Trust 1018 (WT098051 and WT091310), the EU FP7 (EPIGENESYS 257082 and BLUEPRINT 1019 HEALTH-F5-2011-282510). J.A.T is supported by the supported by the Wellcome Trust 
1020 (091157) and JDRF (9-2011-253). K.S. is funded by the Biomedical Research Program funds at Weill Cornell Medicine in Qatar, a program funded by the Qatar Foundation. J.D. is a

1022 British Heart Foundation Professor, European Research Council Senior Investigator, and 1023 National Institute for Health Research (NIHR) Senior Investigator. The INTERVAL study is 1024 funded by NHSBT (11-01-GEN) and has been supported by the NIHR-BTRU in Donor 1025 Health and Genomics (NIHR BTRU-2014-10024) at the University of Cambridge in partnership with NHSBT. The views expressed are those of the authors and not necessarily those of the NHS, the NIHR, the Department of Health of England, or NHSBT.

\section{Author Contributions}

Conceptualization and experimental design: J.D., A.S.B., B.B.S., H.R., R.M.P.; Methodology: B.B.S., A.B.S., J.C.M., J.E.P., H.R., S.B.; Analysis: B.B.S., J.C.M., J.E.P., D.S., J.B., J.R.S., T.J., E.P., P.S., C.O-W., M.A.K., S.K.W., A.C., N.B., S.L.S.; Contributed reagents, materials, protocols or analysis tools: N.J., S.K.W., E.S.Z., J.B., M.A.K., J.R.S., B.P.P.; Supervision: A.S.B., H.R., C.S.F., J.D., R.M.P., D.S.P., A.M.W.; Writing - principal: B.B.S., A.S.B., J.E.P., J.C.M., H.R., J.D.; Writing - review and editing: B.B.S., A.S.B., J.E.P., J.C.M., J.D., H.R., K.S., A.M.W., N.J., D.J.R., J.A.T., D.S.P., N.S., C.S.F., R.M.P; Creation of the INTERVAL BioResource: J.R.B., D.J.R., W.H.O., N.W.M., J.D.; Funding 1037 acquisition: N.W.M., J.R.B., D.J.R., W.H.O., C.S.F., R.M.P., J.D.; all authors critically 1038 reviewed the manuscript.

\section{Author Information}

1040 Reprints and permissions information is available at www.nature.com/reprints. The authors

1041 declare the following competing financial interests: J.C.M., A.C., C.S.F., R.M.P., H.R. are employees at MRL, Merck \& Co., Inc. S.K.W., E.S.Z., N.J. are employees and stakeholders in SomaLogic, Inc. The other authors have nothing to disclose. Correspondence and requests 
bioRxiv preprint doi: https://doi.org/10.1101/134551; this version posted May 5, 2017. The copyright holder for this preprint (which was not

certified by peer review) is the author/funder. All rights reserved. No reuse allowed without permission.

1044 for materials should be addressed to A.S.B. (asb38@medschl.cam.ac.uk) and J.D.

1045 (jd292@medschl.cam.ac.uk).

1046 


\section{Figures}

1048 Figure 1. The genetic architecture of plasma protein levels.

1049 (a) Genomic location of pQTLs. Plot of sentinel variants for pQTLs (red=cis, blue= trans).

1050 Y-axis indicates the position of the gene that encodes the associated protein. The twelve most

1051 associated regions of the genome are annotated. (b) Plot of the statistical significance of the

1052 most associated (sentinel) cis variant for each protein against the distance from the

1053 transcription start site (TSS). (c) Histogram of the number of significantly associated loci per

1054 protein. (d) Histogram of the number of conditionally significant signals within each

1055 associated locus. (e) Histogram of protein variance explained (adjusted $\mathrm{R}^{2}$ ) by conditionally

1056 significant variants. (f) Distribution of effect size against minor allele frequency (MAF) for

1057 cis and trans pQTLs. (g) Distribution of the predicted consequences of the sentinel pQTL

1058 variants compared to matched permuted null sets of variants. Asterisks highlight empirical

1059 enrichment $p<0.005$. 
a

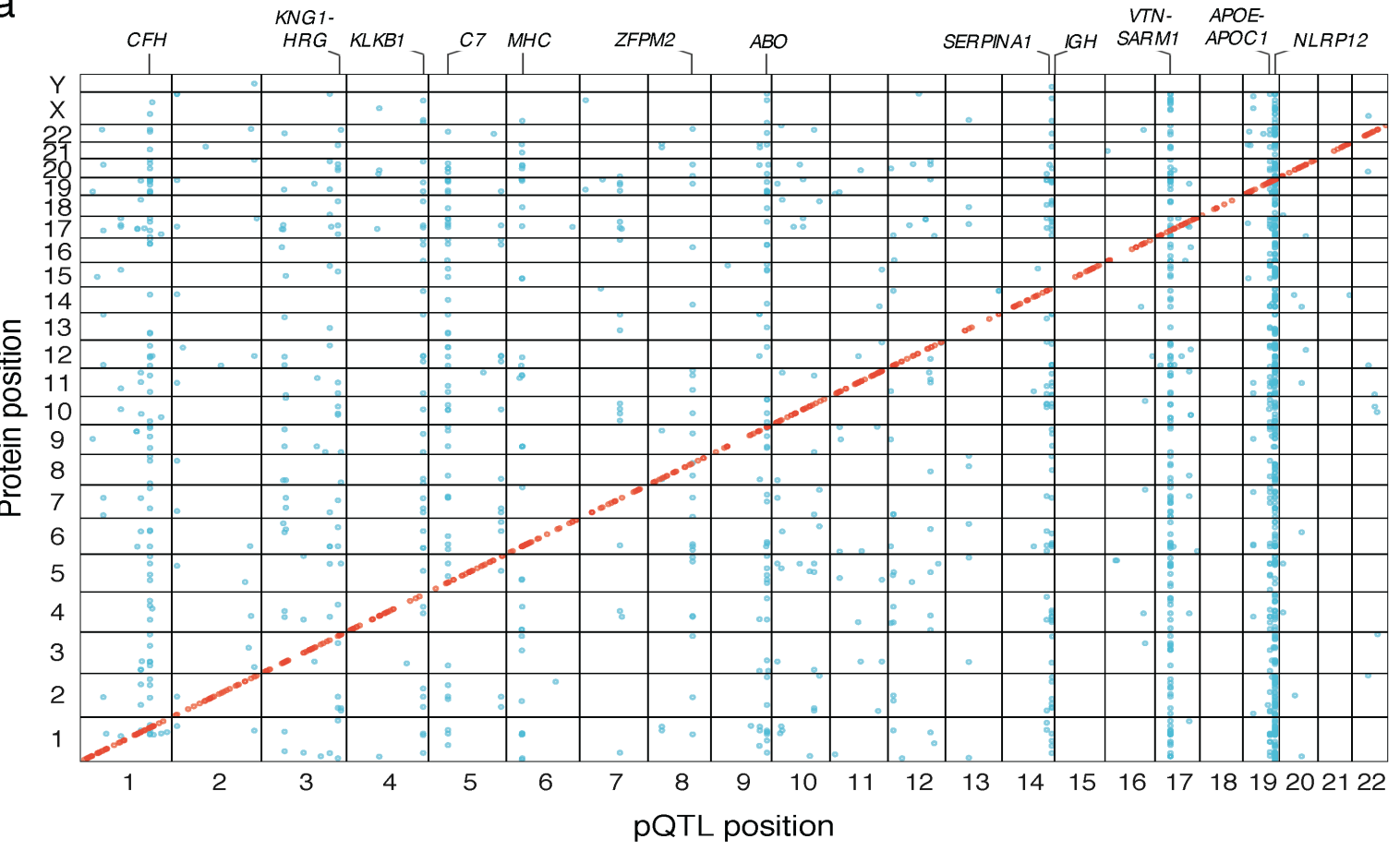

b

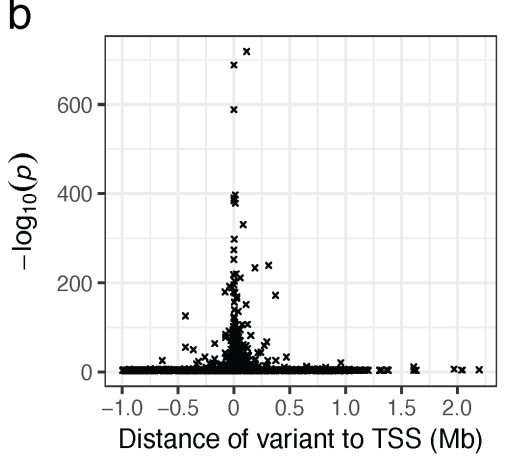

e

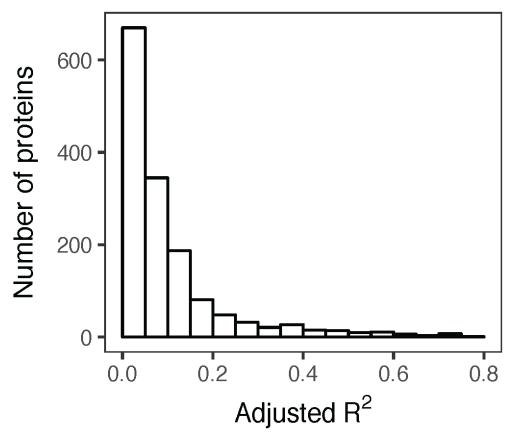

C

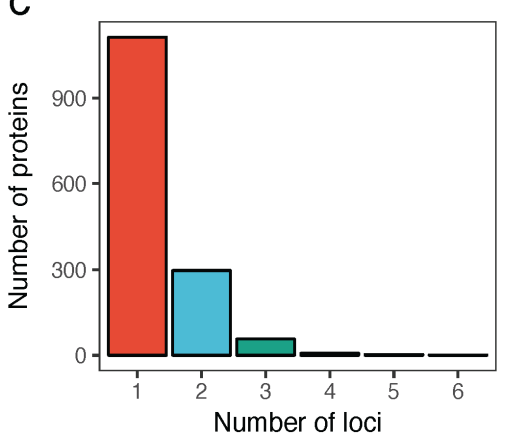

$f$

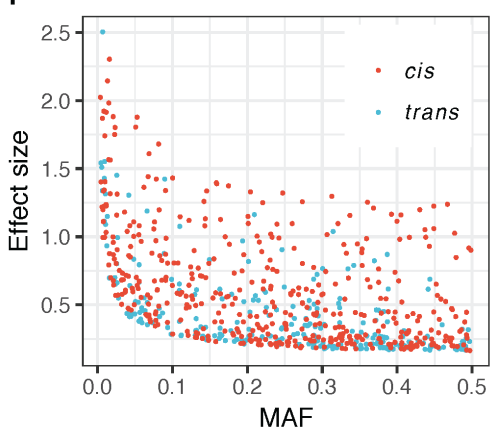

d

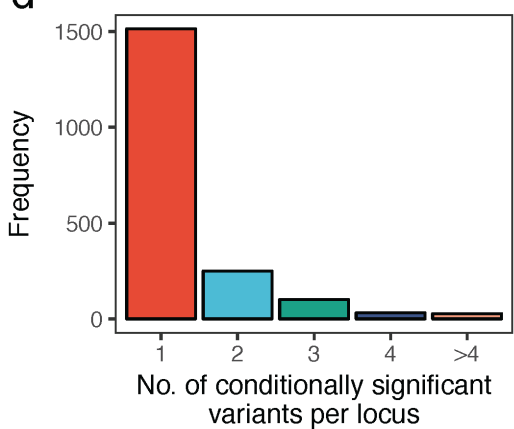

g

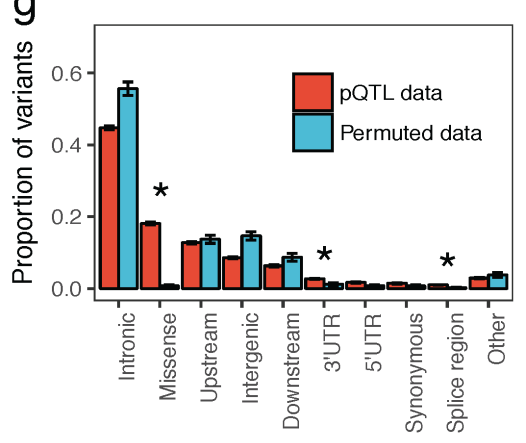


1062 Figure 2. The GDF11/8 trans pQTL is mediated by genetic control of WFIKKN2 levels.

1063 (a) Regional association plots of the trans pQTL (sentinel variant rs11079936) for GDF11/8

1064 before and after adjusting for levels of WFIKKN2 (upper panels), and the WFIKKN2 cis

1065 pQTL after adjusting for GDF11/8 levels (bottom panel). A similar pattern of association for

1066 WFIKKN2 was seen prior to GDF11/8 adjustment (not shown). (b) Proposed mechanism of

1067 how the trans pQTL for GDF11/8 is mediated by WFIKKN2 levels.

Chr 17

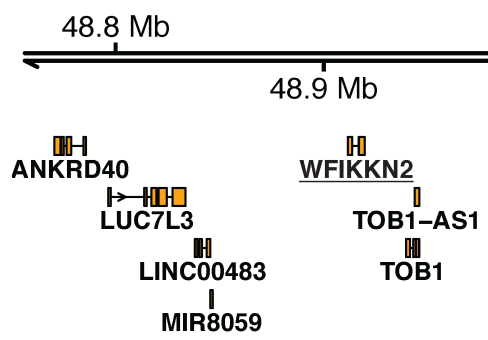

| rs11079936

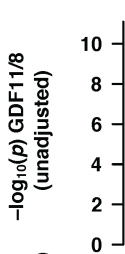

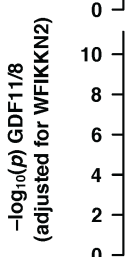

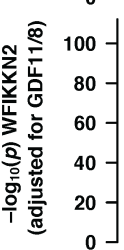

$\because$

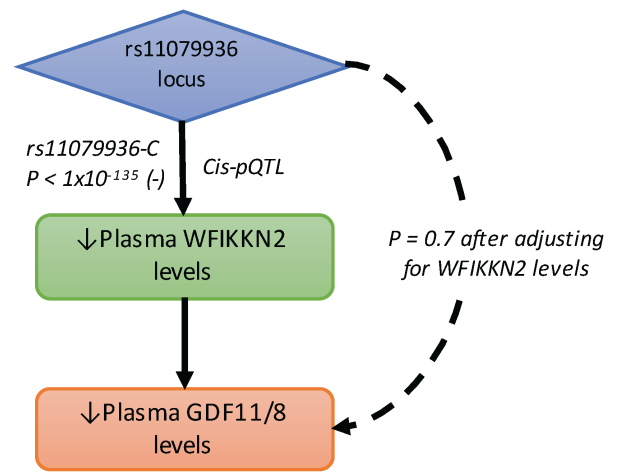

levels

1068
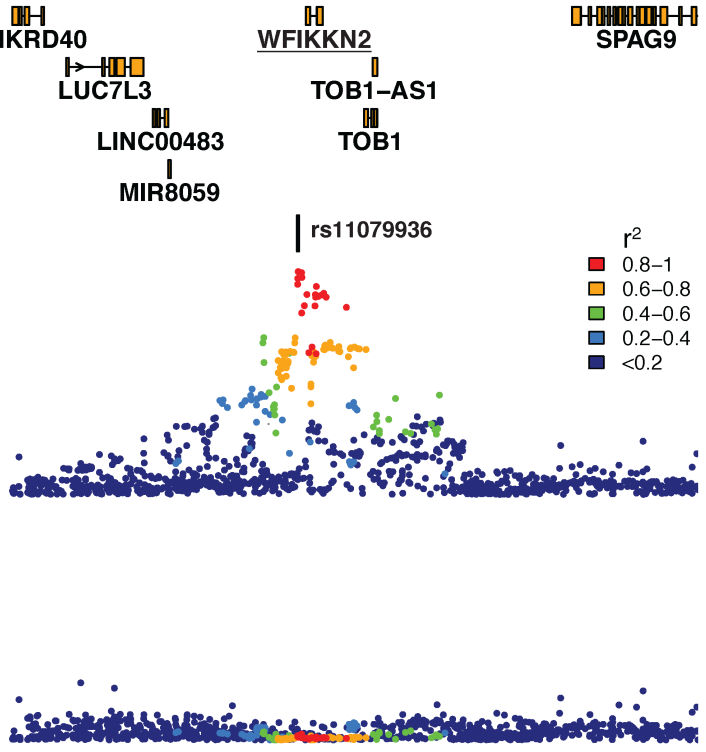

$0.4-0.6$

- $<0.2$

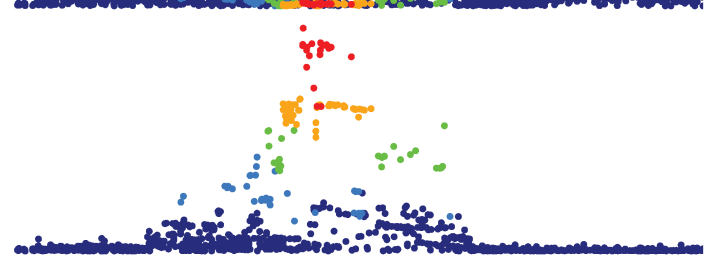


1072 (a) Missense variant rs3197999 in the MST1 region on chromosome 3 is associated with IBD

1073 (top) and BLIMP1 levels (bottom). (b) Regional association plot of the IBD susceptibility 1074 locus on chromosome 6 adjacent to the PRDM1 gene, which encodes BLIMP1. IBD association data are for European participants from Liu et al., 2015. (c) Boxplot for relative 1076 plasma BLIMP1 levels by rs3197999 genotype, stratified by the two subcohorts used in our 1077 analysis.

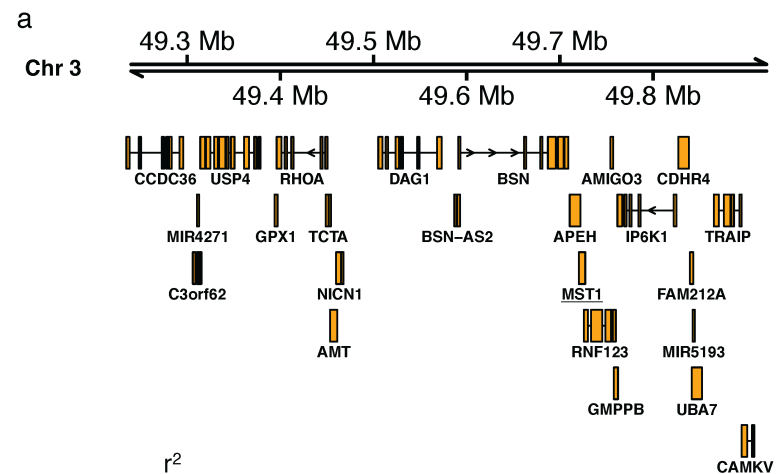

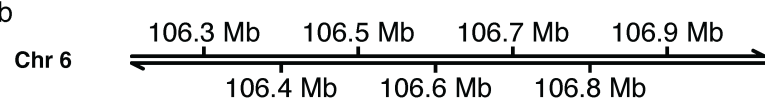
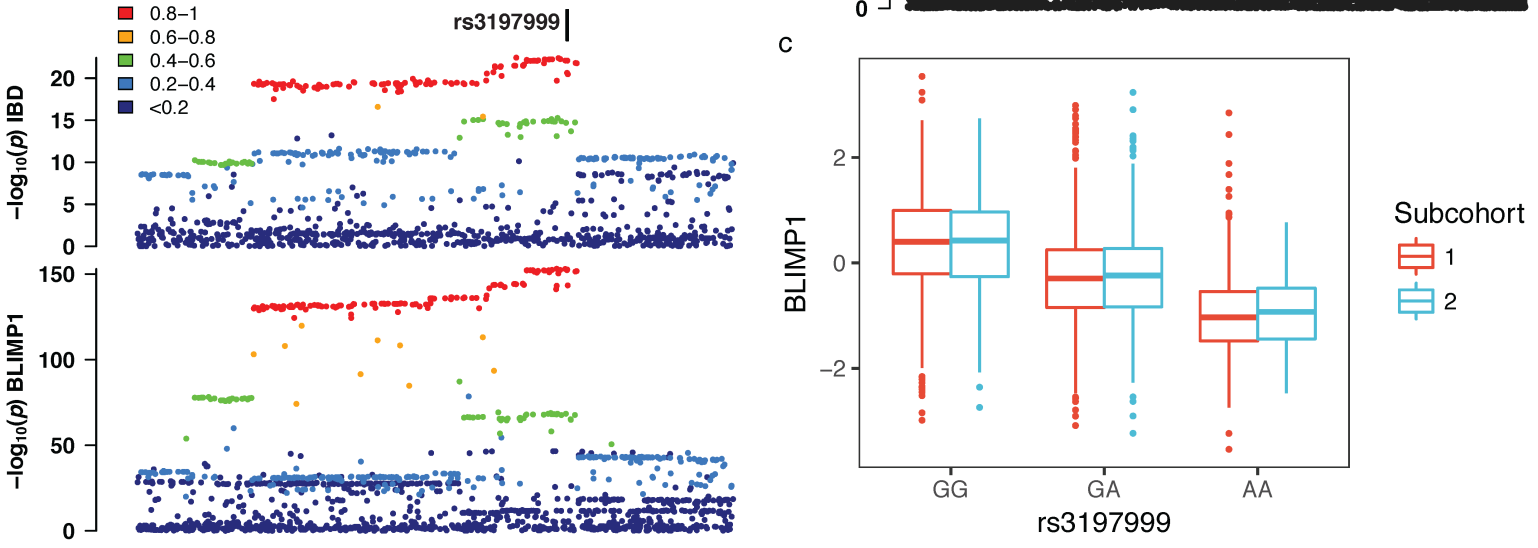
Figure 4. Missense variant rs28929474 in SERPINA1 is a trans pQTL hotspot.

1081 Numbers (outermost) indicate chromosomes. Interconnecting lines link the genomic location

1082 of rs 28929474 and the genes encoding significantly associated $\left(p<1.5 \times 10^{-11}\right)$ proteins. Line

1083 thickness is proportional to the effect size of the associations with red positive and blue

1084 negative.

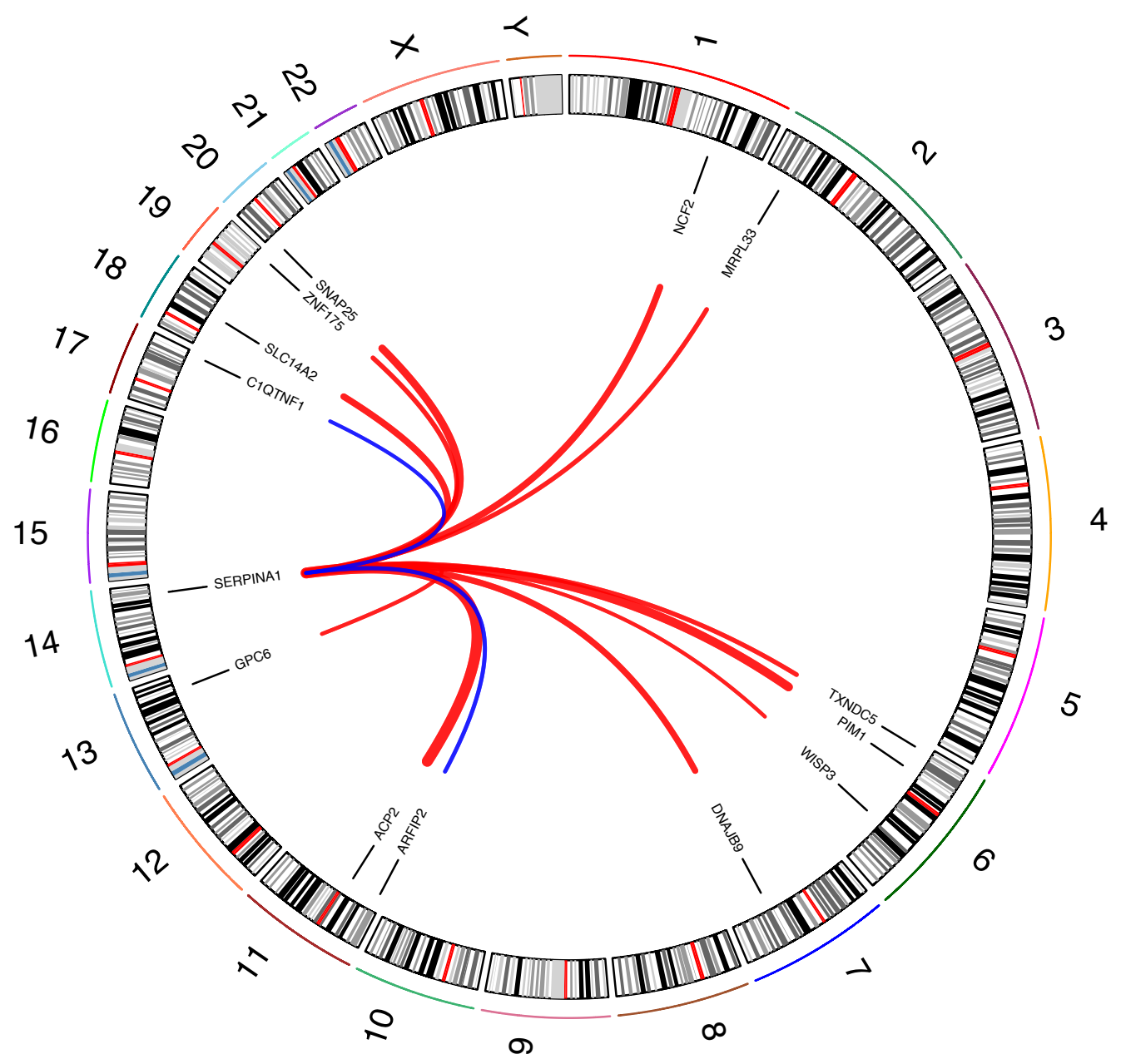


1088 Figure 5. Evaluation of causal role of proteins in disease.

1089 Forest plot of univariate and multivariable Mendelian randomization (MR) estimates. (a)

1090 Proteins in the ILIRL1-IL18RI locus and risk of atopic dermatitis (AD). No univariate MR

1091 estimates available for IL1R1 and IL18RAP due to no significant pQTLs to select as a

1092 "genetic instrument". (b) MMP-12 levels and risk of coronary heart disease (CHD).

1093

a

Protein

IL18R1

Univariate MR

Multivariable MR

IL1RL1

Univariate MR

Multivariable MR

IL1RL2

Univariate MR

Multivariable MR

IL1R2

Univariate MR

Multivariable MR

IL1R1

Multivariable MR

IL18RAP

Multivariable MR

$\mathrm{b}$

MMP12

Univariate MR

Multivariable MR
Higher levels decrease risk

Higher levels increase risk

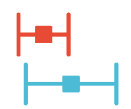

$9.3 e-72$

$1.5 e-28$

5.7e-27

0.01

0.013

$1.1 \mathrm{e}-69$

0.7

0.0094

0.49

0.97

$\begin{array}{lllllll}-0.3 & -0.2 & -0.1 & 0 & 0.1 & 0.2 & 0.3\end{array}$

Effect on $A D$ risk per unit change in protein levels

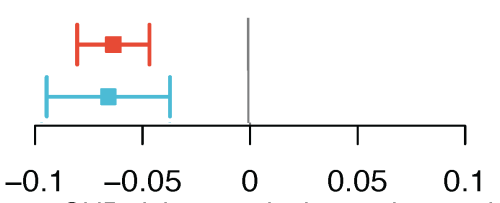

Effect on $\mathrm{CHD}$ risk per unit change in protein levels
P-Value

$2.8 \mathrm{e}-13$

$8.6 e-06$ 